\title{
An Unusually Late Presentation of Recurrent Cardiac Syncope in Kearns Sayre Syndrome
}

Dinushi Dilanka Dikowita ${ }^{\text {* }}$, Thirunavukarasu Kumanan², Muthusamy Malaravan ${ }^{3}$ and Mahesan Guruparan ${ }^{4}$

${ }^{1}$ Department of Medicine, Teaching Hospital Jaffna, Sri Lanka

${ }^{2}$ Department of Medicine, Faculty of Medicine, Jaffna, Sri Lanka

${ }^{3}$ Department of Ophthalmology, Teaching Hospital Jaffna, Sri Lanka

${ }^{4}$ Department of Cardiology, Teaching Hospital Jaffna, Sri Lanka

\begin{abstract}
Background: Kearns Sayre Syndrome (KSS) is a rare mitochondrial disorder with systemic involvement characterised by skeletal, smooth and cardiac muscle dysfunction; pigmentary retinal changes; neurological and endocrine dysfunction. KSS arise from a large-scale deletion of mitochondrial DNA which typically occurs as a sporadic event. Recurrent cardiac syncope is one way of the initial presentation of this syndrome due to involvement of cardiac conduction system.
\end{abstract}

Case presentation: A 51-year-old male, previously well apart from having stable angina for which he was on aspirin and statin, presented to medical casualty with recurrent syncopal episodes with loss of consciousness lasting 3 minutes to 5 minutes and spontaneous recovery after each event. On ocular examination he was found to be have poor vision (best corrected visual acuity in both eyes was 6/60), bilateral posterior subcapsular cataract and both eyes fundus revealed features of retinitis pigmentosa with cystoid macular oedema. A 12 lead electrocardiography (ECG) on admission showed evidence of atrial flutter with variable heart block leading to significant pauses and the patient underwent permanent pacemaker insertion as he was severely symptomatic. Patient was referred to visual rehabilitation.

Conclusion: Mitochondrial myopathy is an important differential diagnosis which needs to be considered in a patient presenting with recurrent cardiac syncope in the context of multisystem involvement.

Keywords: Cardiac syncope; Kearns Sayre syndrome

\section{Introduction}

Mitochondria are the intracellularorganellesinvolved in maintaining the aerobic energy metabolism with high yield of ATP production, in order to fulfil the energy requirements of the cell. Therefore any primary dysfunction of the respiratory chain (Krebs cycle) which occurs inside the mitochondria will jeopardize the energy homeostasis of individual cell hence ultimately affecting all metabolically active tissues including muscle tissue. Kearns-Sayre syndrome is a mitochondrial myopathy with widespread multisystem involvement featured by onset before the age of 20, chronic progressive external opthalmoplegia, pigmentary retinopathy and cardiac conduction defects.

We describe here a 51-year-old male who presented with recurrent cardiac syncopal episodes with electrocardiographic evidence of life threatening arrhythmias with conduction abnormalities, and simultaneous finding of marked low vision due to pigmentary retinopathy in the same patient suggesting possible systemic disease such as mitochondrial myopathy.

\section{Case Presentation}

A 51-year-old male presented to the medical casualty ward with a history of recurrent syncopal episodes over the last 4 months duration. Each episode was preceded by chest discomfort, palpitations and syncopal episode with loss of consciousness for less than five minutes. During each episode he regained consciousness spontaneously and had no neurological deficit afterwards. On further questioning he revealed that he has lost his vision for the last three years and he is dependant on his siblings for day to day activities. Apart from stable angina, for which he was on aspirin $150 \mathrm{mg}$ and atorvastatin $20 \mathrm{mg}$ daily; his past medical history was unremarkable. On examination he was a short statured person $(154 \mathrm{~cm})$. Radial pulse on admission was irregular; however he was haemodynamically stable. His 12 lead ECG on admission showed atrial flutter with variable block (Figure 1).

Troponin I was normal on admission $(0.037 \mathrm{ng} / \mathrm{ml}(<0.15 \mathrm{ng} / \mathrm{ml}))$ and a $2 \mathrm{D}$ echocardiography done revealed reasonably good systolic and diastolic left ventricular function (ejection fraction 60\%) with thickened mitral valve; grade 111 mitral regurgitation. Following day he underwent 24 hour holter monitoring and it revealed short runs of Ventricular tachycardia (VT) with significant pauses (Figure 2).

Patient underwent single chamber pacemaker insertion in VVI mode under local anaesthesia and backup rate was set at $60 / \mathrm{min}$ and planned for further cardiology follow-up. His ophthalmological assessment further confirmed low vision with best corrected visual acuity (6/60 and 6/60) and revealed evidence of bilateral retinitis pigmentosa (Figure 3). Patient was followed up at ophthalmology clinic and referred to visual rehabilitation.

\section{Discussion and Conclusion}

Kearns-Sayre Syndrome (KSS) is a rare mitochondrial myopathy with heterogeneous clinical presentation. The classical triad of the syndrome includes external opthalmoplegia, pigmentary retinopathy

*Corresponding author: Dinushi Dilanka Dikowita, Department of Medicine, Teaching Hospital Jaffna, Sri Lanka, Tel: +94 212 223348; E-mail: dinushidilankadikowitadikowita@yahoo.com

Received June 25, 2017; Accepted July 31, 2017; Published August 07, 2017

Citation: Dikowita DD, Kumanan T, Malaravan M, Guruparan M (2017) An Unusually Late Presentation of Recurrent Cardiac Syncope in Kearns Sayre Syndrome. J Cardiovasc Dis Diagn 5: 289. doi: 10.4172/2329-9517.1000289

Copyright: (c) 2017 Dikowita DD, et al. This is an open-access article distributed under the terms of the Creative Commons Attribution License, which permits unrestricted use, distribution, and reproduction in any medium, provided the original author and source are credited. 

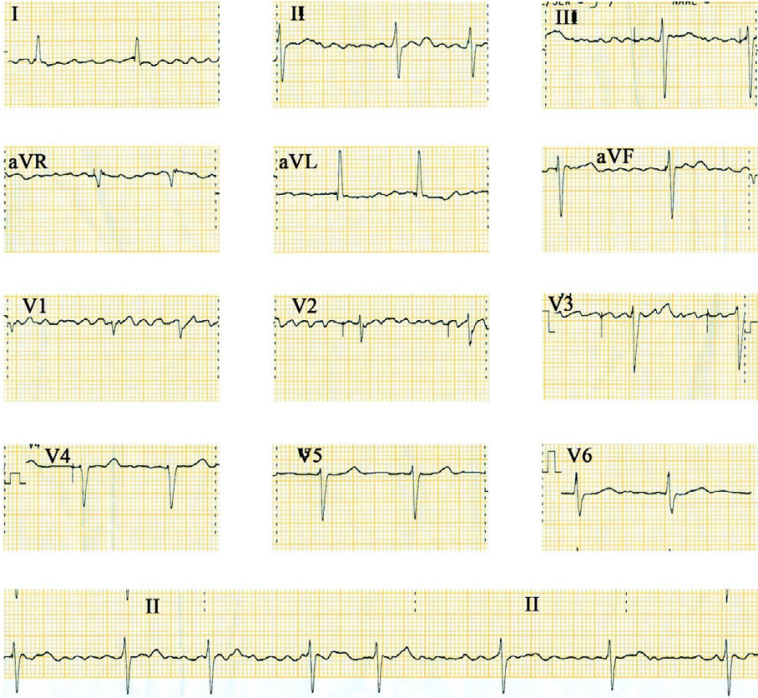

Figure 1: Electrocardiograph on admission showing atrial flutter with variable block.

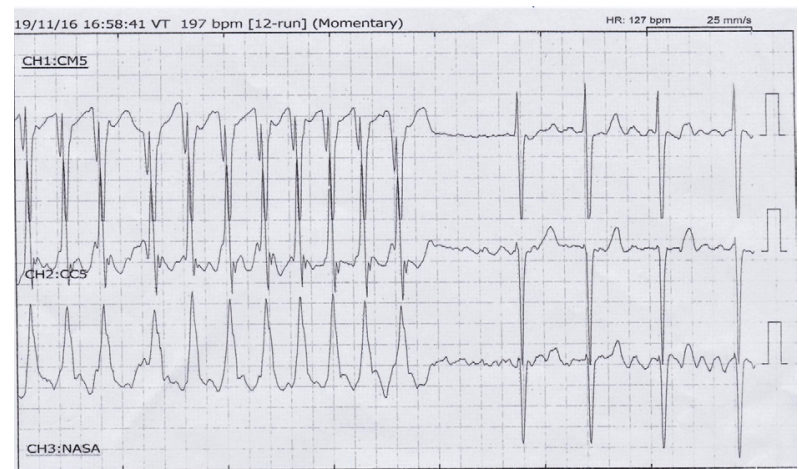

Figure 2: Holter tracing showing short runs of VT with significant pauses.

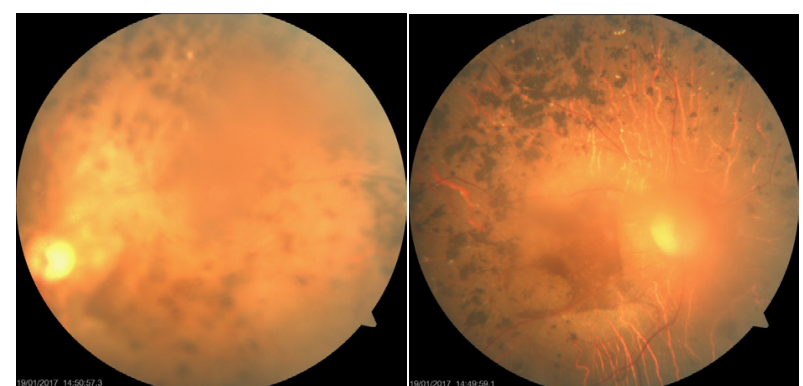

Figure 3: Left and right fundus photographs of the patient showing retinal and macular changes.

and progressive degeneration of cardiac conduction system usually before the age of 20 [1]. The diagnostic features of KSS consist of progressive external ophthalmoplegia, onset before age 20 with pigmentary retinopathy together with one of the following; cerebrospinal fluid protein greater than $1 \mathrm{~g} / \mathrm{L}$, cerebellar ataxia or heart block [2]. The clinical diagnosis of Kearns Sayre Syndrome among the adult population is a difficult task due to the clinical heterogeneity of this condition, however genetic testing and muscle biopsy will provide definitive evidence in high resource setting. This case highlights the importance of considering a multisystem disease as a diagnosis rather than considering isolated heart and eye involvement in arriving at final diagnosis. Though there are case reports highlighting KSS presenting as cardiac conduction abnormalities in younger age group, our case highlight that it can also be a cause of recurrent cardiac syncope in middle age population particularly in the presence of multiple organ system involvement. This fact is also in line with the findings discussed by Khambatta et al. [3] in their case series on KSS based on Mayo clinic medical index patient database between 1976 and 2009, where they also reiterate though the existing diagnostic criteria require that patients have symptom onset before 20 years, $20 \%$ of the patients included in their study, were more than 20 years at presentation [3]. In addition to the age criteria, this patient fulfils one of the typical eye changes of the disease, being pigmentary retinopathy and echocadiographic evidence of heart block fulfilling the minimal criteria to diagnose KSS.

Eye involvement in mitochondrial myopathy is characteristic. Extra ocular muscles invariably have high energy requirement and mitochondria make up approximately $60 \%$ of total cell volume of extraocular muscles [3], explaining the above statement. Ophthalmologic manifestations of KSS have wide range of clinical presentation. Chronic progressive opthalmoplegia is the most frequent manifestation followed by bilateral symmetrical ptosis. Our patient doesn't have these features. While the typical retinal changes include pigmentary degeneration causing low visual acuity, certain subset of patients develop salt and pepper or moth eaten appearance characterised by diffuse depigmentation of retinal epithelium which will be more evident near macula [4]

Cardiac involvement is frequent in KSS and it is a major determinant of prognosis of these patients. The clinical manifestations of heart disease in KSS include cardiac syncope (45\%), sudden death (23\%) and cardimyopathy (20\%) [5]. Further mitral and tricuspid valve lesions are also established findings in heart disease in KSS. With regard to this patient, though he was an ideal candidate for ICD insertion, in the scarcity of adequate facilities as a bridging therapy he underwent PPM insertion. However there is no evidence for prophylactic permanent pacemaker or ICD insertion in these patients unless there is strong clinical, echocardigraphic or holter evidence of life threatening arrhythmias.

\section{Acknowledgements}

None

\section{Competing Interests}

The authors declare that they have no competing interests.

\section{Consent for Publication}

Written informed consent was obtained from the patient for publication of this case report and accompanying images.

\section{Ethics Approval and Consent to Participate}

Not applicable.

\section{References}

1. Gobu P, Karthikeyan B, Prasath A, Santhosh S, Balachander J (2010) Kearns Sayre Syndrome (KSS)-A rare cause for cardiac pacing. Indian Pacing Electrophysiol J 10: 547 .

2. Warral DA, Cox TM (2003) Oxford Textbook of Medicine, (4th edn), Oxford University Press, USA.

3. Khambatta S, Nguyen DL, Beckman TJ, Wittich CM (2014) Kearns-Sayre syndrome: A case series of 35 adults and children. Int J Gen Med 7: 325.

4. Ahmad SS, Ghani SA (2012) Kearns-Sayre syndrome: An unusual ophthalmic presentation. Oman J Ophthalmol 5: 115-117.

5. Barrera-Ramírez CF, Barragán-Campos HM, Ilarraza $H$, Iturralde $P$, Ávila Casado MC et al. (2005) Cardiac involvement in Kearns-Sayre Syndrome. Rev Esp Cardiol 58: 443-446. 